\title{
Variational Iteration Method and Sumudu Transform for Solving Delay Differential Equation
}

\author{
Subashini Vilu $(\mathbb{D}$, Rokiah Rozita Ahmad $\mathbb{D}$, and Ummul Khair Salma Din \\ School of Mathematical Sciences, Faculty of Science \& Technology, Universiti Kebangsaan Malaysia, \\ 43600 UKM Bangi, Selangor, Malaysia \\ Correspondence should be addressed to Rokiah Rozita Ahmad; rozy@ukm.edu.my
}

Received 17 January 2019; Accepted 25 March 2019; Published 2 May 2019

Academic Editor: Elena Kaikina

Copyright (C) 2019 Subashini Vilu et al. This is an open access article distributed under the Creative Commons Attribution License, which permits unrestricted use, distribution, and reproduction in any medium, provided the original work is properly cited.

In this research, a new approach is presented for solving delay differential equations (DDEs) which is a blend of Sumudu transform and variational iteration method (VIM). A general Lagrange multiplier is used to construct a correction functional. This is done with an uncommon Sumudu transform alongside variational theory. A few numerical cases were solved to demonstrate methodology of this new approach. Objective of this research is to reduce the complexity of computational work compared to the conventional approaches. It can be concluded that the amount of evaluation is reduced but at the same time the results are comparable as in the previous works.

\section{Introduction}

In recent years, especially, for several decades, applications of powerful tools for solving numerical and analytical cases have attracted attentions of scientists from all over the world [1]. Delay differential equations (DDEs) are a kind of functional differential equation having a widespread range of uses in the arena of science, technology, and engineering which acquires numerical/analytical solutions. It had already been applied in control theory for many years and recently it is being used extensively in many biological models. DDEs are form of differential equation in which the derivative of the unknown function at a particular period is provided in terms of values of the function at earlier periods. Introducing delays in models enhances the vitality of these models and allows an accurate depiction of actual occurrences. DDEs arise commonly in various physical occurrences. To be specific, they are essential once ordinary differential equation (ODE) based models are unsuccessful. In disparate ODEs, where initial conditions are stated at preliminary point, DDEs need history of the system over the delayed interval which are then provided as initial conditions. In line with this, delay systems turn out to be intricate and multifaceted in nature which in turn complicates the process of analyzing DDEs analytically and therefore requires a numerical approach. The delay differential equation in its simplest form is

$$
v_{i}^{\prime}(t)=f\left(t, v_{i}(t), v_{i}(q t)\right)
$$

in which $i=1,2,3, \ldots, n$ and $q$ is a constant delay.

Application of a few numerical methods that were introduced turns out to be very useful to solve these types of equations as in numerical simulations such as the variational iteration method and the optimal perturbation method. DDE problems have constantly led to an infinite spectrum of frequencies. Hence, few approaches have been implemented in solving them such as approximation, asymptotic solutions, numerical method, and graphical approaches. In recent times, Anakira et al. employed the Optimal Homotopy Asymptotic Method (OHAM) in solving linear and nonlinear DDE [2] while Alomari et al. used the Homotopy Analysis Method (HAM) to find the solution for DDE [3]. Among all these methods applied to solve differential equations, one of them is the variational iteration method (VIM) which was firstly initiated by Ji-Huan He which could be seen throughout [4-8]. Subsequent works [9-17] reflect the flexibility, consistency, and effectiveness of the procedure in VIM. The application of VIM to differential equations generally involves discovering a correction functional, finding the Lagrange multiplier and deciding on a good initial 
approximation. Application of VIM has remained successful on initial as well as boundary value problems, Schrodinger equations, integrodifferential equation, fractional differential equation, chaotic Chen system, coupled sine-Gordon equation, general Riccati differential equations, fractional heatand wave-like equations, and many more.

Various authors have made efforts in the developments of the VIM with the wide application of the method. Particularly, Wu $[13,16]$ used the Laplace transform to compute the Lagrange multipliers, which overcomes main shortcomings in implementation of the VIM to fractional equations. Sumudu transform is a simple variant of the Laplace transform and is essentially identical with the Laplace. Sumudu transform has many interesting properties that make it easy to visualize making it an ideal transform for control engineering and applied mathematicians. Recently, Sumudu transform is implemented in some well-known analytical approaches [19], where the coupling of homotopy perturbation method (HPM) and Sumudu transform is used to make the process of the solution simpler and improves the solution's accurateness.

A new modified variational iteration method was found, inspired and driven by Wu's thoughts and combining with the Sumudu transform [20]. The new approach is based on variational iteration theory and Sumudu transform. In this paper, the basic motivation is extending this new reliable approach for the solution of linear and nonlinear DDEs which are normally challenging to analyze due to their multifaceted nature and boundless dimensionality.

\section{Sumudu Variational Iteration Method (SVIM)}

First, let us take the general nonlinear differential equation [21] to illustrate main idea of VIM for DDEs,

$$
\frac{d^{m} v(t)}{d t^{m}}+R[v(t)]+N[v(t)]=f(t)
$$

with the following initial conditions:

$$
v^{(k)}(0)=v_{0}^{k}, \quad k=0,1,2, \ldots,(m-1)
$$

in which $v=v(t), R$ is a linear operator, $N$ is a nonlinear operator, $f(t)$ is a knowncontinuous function, and $d^{m} v(t) / d t^{m}$ is the term of the maximum order derivative.

Basic idea of the VIM is building a correction functional for (2) of formula

$$
\begin{aligned}
& v_{(n+1)}(t) \\
& =v_{n}(t) \\
& \quad+\int \lambda(t, \tau)\left(\frac{d^{m} v_{n}}{d t^{m}}+R\left(\widetilde{v}_{n}\right)+N\left(\widetilde{v}_{n}\right)-f(\tau)\right) d \tau .
\end{aligned}
$$

The successive approximation $v_{n}, n \geq 1$, could be attained by finding $\lambda(\tau)$, a general Lagrange multiplier that could be known optimally with variational theory. The function $\widetilde{v}_{n}$ is considered as a restricted variation indicating $\delta \widetilde{v}_{n}=0$. At first, integration by parts is done to determine the Lagrange multiplier which enables the consecutive approximations, $v_{n}(t)$ of the exact solution $v(t)$ to be attained by means of a good initial approximation $v_{0}(t)$. Initial conditions in (3) typically give the initial approximation. Finally, as $n \longrightarrow$ $\infty, v_{n}(t)$ converges to the exact solution $v(t)$.

2.1. Combination of VIM and Sumudu Transform (SVIM). The entire procedure of Lagrange multipliers is expressed as a case of algebraic equation where its solution $f(x)=0$ could be found by

$$
x_{n+1}=x_{n}+\lambda f\left(x_{n}\right) .
$$

Optimality condition for the extreme $\delta x_{n+1} / \delta x_{n}=0$ advances to

$$
\lambda=-\frac{1}{f^{\prime}\left(x_{n}\right)},
$$

in which $\delta$ represents the traditional variational operator. Implementing the initial point $x_{0}$ provided, the approximate solution $x_{n+1}$ could be determined via next iterative scheme, with (5) and (6)

$$
x_{n+1}=x_{n}-\frac{f\left(x_{n}\right)}{f^{\prime\left(x_{n}\right)}}, \quad f^{\prime}\left(x_{0}\right) \neq 0, n=0,1,2, \ldots
$$

The formula above (7) is the famous Newton-Raphson formula that possesses a quadratic convergence.

In this article, we outspread the idea in finding the unknown Lagrange multiplier. Key step is the application of Sumudu transform into (2) with its fundamental properties in [22]. Then, the linear equation will be converted into an algebraic equation below:

$$
\begin{gathered}
u^{-m} \bar{v}(s)-u^{-m} v(0)-\ldots-u^{-1} v^{m-1}(0) \\
+\mathbb{S}(R[v]+N[v])=\mathbb{S}(f(t))
\end{gathered}
$$

Hence, the algorithm of the Sumudu Variational Iteration Method (SVIM) is given below:

(1) Applying Sumudu transform to (2) gives the correction functional as

$$
\begin{gathered}
\bar{v}_{n+1}(u)=\bar{v}_{n}(u)+\lambda(u)\left[u^{-m} \bar{v}(u)-u^{-m} v(0)-\ldots\right. \\
\left.-u^{-1} v^{m-1}(0)+\mathbb{S}(R[v]+N[v])-\mathbb{S}(f(t))\right]
\end{gathered}
$$

with the notation used $\mathbb{S}$ to indicate Sumudu transform.

(2) Considering the terms $\mathbb{S}\left(R\left(\widetilde{v}_{n}\right)+N\left(\widetilde{v}_{n}\right)\right)-\mathbb{S}(f(t))$ as restricted variations, we let (9) be stationary with respect to $\bar{v}_{n}$

$$
\delta \bar{v}_{n+1}(u)=\delta \bar{v}_{n}(u)+\lambda(u)\left(\frac{1}{u^{m}} \delta \bar{v}_{n}(u)\right) .
$$

From (9), we define Lagrange multiplier as

$$
\lambda(u)=-u^{m}
$$


(3) Succeeding approximations can then be attained with the application of inverse Sumudu transform $\mathbb{S}^{-1}$ into (8) which gives

$$
\begin{aligned}
v_{n+1} & (t)=v_{n}(t)-\mathbb{S}^{-1}\left[u ^ { m } \left\{u^{-m} \bar{v}(u)-u^{-m} v(0)-\ldots\right.\right. \\
- & \left.\left.u^{-1} v^{m-1}(0)+\mathbb{S}\left(R\left(v_{n}\right)+N\left(v_{n}\right)\right)-\mathbb{S}(f(t))\right\}\right], \\
= & \mathbb{S}^{-1}\left(v(0)+\ldots+u^{m-1} v^{m-1}(0)\right) \\
+ & \mathbb{S}^{-1}\left[u^{m}\left(\mathbb{S}\left[R\left(v_{n}\right)+N\left(v_{n}\right)-f(t)\right]\right)\right],
\end{aligned}
$$

with initial approximation

$$
\begin{aligned}
v_{0}(t) & =\mathbb{S}^{-1}\left(v(0)+\ldots+u^{m-1} v^{m-1}(0)\right) \\
& =v(0)+v^{\prime(0)} t+\ldots+\frac{t^{m-1} v^{m-1}(0)}{(m-1) !} .
\end{aligned}
$$

Equation (12) shows that the first iteration in traditional VIM is made up by the Taylor series.

\section{Numerical Applications}

Over this segment, application of the above procedure is done to some nonlinear DDEs.

Example 1. Consider a first order nonlinear DDE

$$
v^{\prime}(t)=1-2 v^{2}\left(\frac{t}{2}\right), \quad v(0)=0 .
$$

The exact solution is known as $v(t)=\sin (t)$.

Taking the Sumudu transform, we obtain

$$
\frac{V(u)}{u}-\frac{v(0)}{u}=\mathbb{S}\left[1-2 v^{2}\left(\frac{t}{2}\right)\right] .
$$

The iteration formula thus is

$$
\begin{aligned}
& V_{n+1}(u) \\
&= V_{n}(u) \\
&+\lambda(u)\left[\frac{V(u)}{u}-\frac{v(0)}{u}-\mathbb{S}\left(1-2 v^{2}\left(\frac{t}{2}\right)\right)\right],
\end{aligned}
$$

and its Lagrange multiplier

$$
\lambda(u)=-u^{m} .
$$

Applying inverse Sumudu transform gives

$$
\begin{aligned}
v_{n+1}(t) & \\
= & v_{n}(t) \\
& -\mathbb{S}^{-1}\left\{u\left[\frac{V(u)}{u}-\frac{v(0)}{u}-\mathbb{S}\left(1-2 v_{n}^{2}\left(\frac{t}{2}\right)\right)\right]\right\}, \\
= & v_{n}(t)+\mathbb{S}^{-1}\left\{u\left[\mathbb{S}\left(1-2 v_{n}^{2}\left(\frac{t}{2}\right)\right)\right]\right\} .
\end{aligned}
$$

TABLE 1: Absolute error comparison representing Example 1.

\begin{tabular}{lcc}
\hline \multicolumn{3}{c}{ Absolute Error } \\
$\mathrm{T}$ & Laplace VIM [18] & Sumudu VIM \\
\hline 0.0 & 0 & 0 \\
0.1 & 0.0980877 & 0.0980877 \\
0.2 & 0.1951794 & 0.1951794 \\
0.3 & 0.290284 & 0.290284 \\
0.4 & 0.3824387 & 0.3824387 \\
0.5 & 0.4707035 & 0.4707035 \\
0.6 & 0.554168 & 0.554168 \\
0.7 & 0.632003 & 0.632003 \\
0.8 & 0.703398 & 0.703398 \\
0.9 & 0.767623 & 0.767623 \\
1.0 & 0.824018 & 0.824018 \\
\hline
\end{tabular}

Therefore,

$$
v_{n+1}(t)=v_{n}(t)+\mathbb{S}^{-1}\left\{u\left[\mathbb{S}\left(1-2 v_{n}^{2}\left(\frac{t}{2}\right)\right)\right]\right\} ;
$$

with initial approximation $v_{0}(t)=0$ and applying the iteration formula (19) above, we attain

$$
\begin{aligned}
v_{1}(t)= & t, \\
v_{2}(t)= & t-\frac{t^{3}}{6}, \\
v_{3}(t)= & t-\frac{t^{3}}{6}+\frac{t^{5}}{120}-\frac{t^{7}}{8064}, \\
v_{4}(t)= & t-\frac{t^{3}}{6}+\frac{t^{5}}{120}-\frac{t^{7}}{5040}+\frac{61 t^{9}}{23224320} \\
& -\frac{67 t^{11}}{3406233600}+\frac{t^{13}}{12881756160} \\
& -0.125146226 \times 10^{-12} t^{15} .
\end{aligned}
$$

From these approximations, it can be seen that the solution tends to form the Taylor series expansion of $\sin (t)$. In order to attest numerically whether or not the suggested approach maintains the accurateness, numerical solutions of the approximation up to $v_{4}$ were evaluated. The absolute values of LVIM and SVIM are compared in Table 1 while Figure 1 displays behavior of the error between these two methods in Example 1. The outcome is in good agreement with each other. Terms of sequences obtained from SVIM are computed using the Maple package.

Example 2. Let us look at second order linear DDE for a second example.

$$
\begin{aligned}
v^{\prime \prime}(t) & =\frac{3}{4} v(t)+v\left(\frac{t}{2}\right)-t^{2}+2, \\
v(0) & =0 \\
v^{\prime}(0) & =0 .
\end{aligned}
$$

The exact solution is given by $v(t)=t^{2}$. 


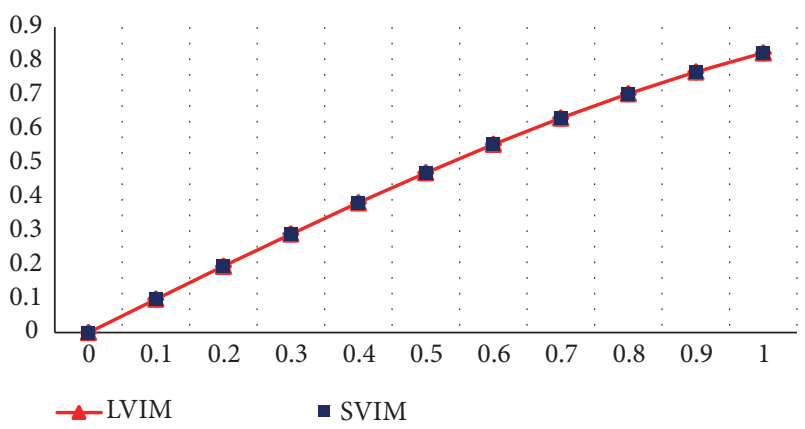

Figure 1: Graphs of the absolute errors for some values of $t$ with LVIM and SVIM for Example 1.

Taking the Sumudu transform, we obtain

$$
\begin{aligned}
& V_{n+1}(u)=V_{n}(u)+\lambda(u)\left[\frac{V(u)}{u^{2}}-\frac{v(0)}{u^{2}}-\frac{v^{\prime}(0)}{u}\right. \\
& \left.\quad-\mathbb{S}\left(\frac{3}{4} v(t)+v\left(\frac{t}{2}\right)-t^{2}+2\right)\right],
\end{aligned}
$$

and its Lagrange multiplier

$$
\lambda(u)=-u^{2}
$$

Applying inverse Sumudu transform gives

$$
\begin{aligned}
& v_{n+1}(t) \\
& =v_{n}(t) \\
& \quad+\mathbb{S}^{-1}\left\{u^{2}\left[\mathbb{S}\left(\frac{3}{4} v(t)+v\left(\frac{t}{2}\right)-t^{2}+2\right)\right]\right\},
\end{aligned}
$$

with initial approximation $v_{0}(t)=0$. Thus, from (24), we attain

$$
\begin{aligned}
& v_{1}(t)=t^{2}-\frac{t^{4}}{12}, \\
& v_{2}(t)=t^{2}-\frac{13 t^{6}}{5760}, \\
& v_{3}(t)=t^{2}-4.09323 \times 10^{-5} t^{8}, \\
& v_{4}(t)=t^{2}-3.428788 \times 10^{-7} t^{10} .
\end{aligned}
$$

The convergence of the approximations to the exact solution $t^{2}$ could be observed from the solution. The absolute values of LVIM and SVIM are compared in Table 2 while Figure 2 displays behavior of error between these two methods in Example 2. The values demonstrate that the outcomes are in excellent agreement with those of the other approaches.
TABLE 2: Absolute error comparison representing Example 2.

\begin{tabular}{lcc}
\hline \multicolumn{2}{c}{ Absolute Error } \\
$\mathrm{t}$ & Laplace VIM [18] & Sumudu VIM \\
\hline 0.0 & 0 & 0 \\
0.1 & 0 & 0 \\
0.2 & 0 & 0 \\
0.3 & 0 & 0 \\
0.4 & 0 & 0 \\
0.5 & $3 \mathrm{E}-10$ & $3 \mathrm{E}-10$ \\
0.6 & $1.6 \mathrm{E}-09$ & $2.1 \mathrm{E}-09$ \\
0.7 & $7.3 \mathrm{E}-09$ & $9.7 \mathrm{E}-09$ \\
0.8 & $2.78 \mathrm{E}-08$ & $3.68 \mathrm{E}-08$ \\
0.9 & $9.01 \mathrm{E}-08$ & $1.196 \mathrm{E}-07$ \\
1.0 & $2.585 \mathrm{E}-07$ & $3.429 \mathrm{E}-07$ \\
\hline
\end{tabular}

Example 3. Lastly, a third order nonlinear DDE is considered

$$
\begin{aligned}
v^{\prime \prime \prime}(t) & =-1+2 v^{2}\left(\frac{t}{2}\right), \\
v(0) & =0, \\
v^{\prime}(0) & =1, \\
v^{\prime \prime}(0) & =0 .
\end{aligned}
$$

Exact solution is known as $v(t)=\sin (t)$.

Succeeding the processes of earlier examples, we attain the following consecutive approximations:

$$
\begin{aligned}
v_{1}(t)= & t-\frac{t^{3}}{6}+\frac{t^{5}}{120}, \\
v_{2}(t)= & t-\frac{t^{3}}{6}+\frac{t^{5}}{120}-\frac{t^{7}}{5040}+\frac{t^{9}}{362880}-\frac{t^{11}}{45619200} \\
& +7.904061 \times 10^{-11} t^{13} \\
v_{3}(t)= & t-\frac{t^{3}}{6}+\frac{t^{5}}{120}-\frac{t^{7}}{5040}+\frac{t^{9}}{362880}-\frac{t^{11}}{39916800} \\
& +1.605904 \times 10^{-10} t^{13}-7.635961 \\
& \times 10^{-13} t^{15}, \\
v_{4}(t)= & t-\frac{t^{3}}{6}+\frac{t^{5}}{120}-\frac{t^{7}}{5040}+\frac{t^{9}}{362880}-\frac{t^{11}}{39916800} \\
& +1.605904 \times 10^{-10} t^{13}-7.647106 \\
& \times 10^{-13} t^{15}+2.811514 \times 10^{-15} t^{17}
\end{aligned}
$$

That converges to exact solution $v(t)=\sin (t)$ when $n \longrightarrow \infty$. Approximate solutions obtained with SVIM could be seen to have a good agreement with the other method. The absolute values of LVIM and SVIM are compared in Table 3 while Figure 3 displays the behavior of the error among these two methods in Example 3. 


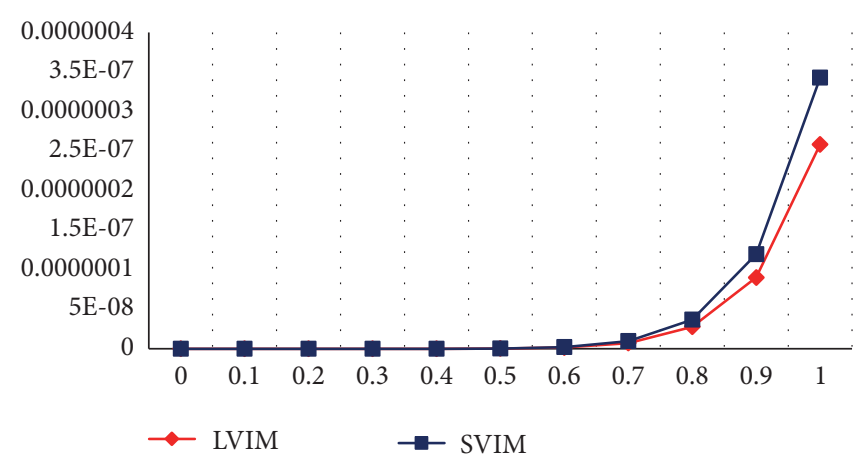

FIGURE 2: Graphs of the absolute errors for some values of $t$ with LVIM and SVIM for Example 2.

TABLE 3: Absolute error comparison representing Example 3.

\begin{tabular}{lcc}
\hline \multicolumn{2}{c}{ Absolute Error } \\
$\mathrm{t}$ & Laplace VIM [18] & Sumudu VIM \\
\hline 0.0 & 0 & 0 \\
0.1 & 0.098088117 & 0.098088117 \\
0.2 & 0.195178731 & 0.195178731 \\
0.3 & 0.290284207 & 0.290284207 \\
0.4 & 0.382437042 & 0.382437042 \\
0.5 & 0.470699039 & 0.470699039 \\
0.6 & 0.554170473 & 0.554170473 \\
0.7 & 0.632000687 & 0.632000687 \\
0.8 & 0.703394091 & 0.703394091 \\
0.9 & 0.76761991 & 0.76761991 \\
1.0 & 0.824018986 & 0.824018985 \\
\hline
\end{tabular}

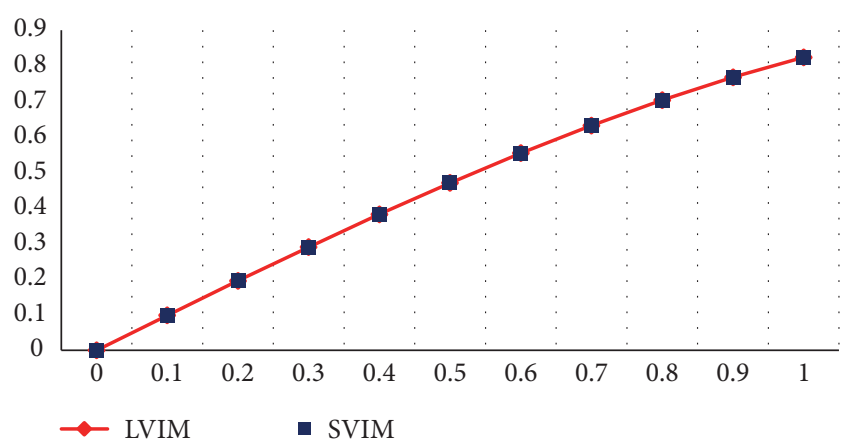

Figure 3: Graphs of the absolute errors for some values of $t$ with LVIM and SVIM for Example 3.

\section{Conclusion}

The Sumudu transform is a simple variant of the Laplace transform and is essentially identical with the Laplace. It has many interesting properties that make it easy to visualize and hence making the process of the solution simpler. SumuduLagrange multiplier is derived from Sumudu transform plus integrating with procedures of VIM to obtain the approximate solutions to DDEs. Lagrange multipliers, $\lambda(u)$ which are defined in (11), could be known optimally with this different approach of variational theory. A new modification of the VIM was attained. This recommended procedure was obtained by not having applied any linearization, discretization, or impractical rules. This new technique provides more convincing or accurate sequence of results that converges quickly in physical problems. In this article, the SVIM was successfully applied in solving linear and nonlinear DDEs. In order to attest numerically whether or not the suggested approach maintains the accurateness, numerical solutions of the approximation up to $v_{4}$ were evaluated. The absolute values of LVIM and SVIM are compared in the tables while Figures 1, 2, and 3 display behavior of the error between these two methods in Example 1 to Example 3. The outcomes are in good agreement with each other. It is worth stating that this method is efficacious in minimizing number of computations contrasting with traditional methods in spite of sustaining great accurateness of the numerical outcome.

\section{Data Availability}

The datasets generated during and/or analysed during the current study are available from the corresponding author on reasonable request.

\section{Conflicts of Interest}

The authors declare that they have no conflicts of interest.

\section{References}

[1] N. R. Anakira, A. Jameel, A. K. Alomari, A. Saaban, M. Almahameed, and I. Hashim, "Approximate Solutions of MultiPantograph Type Delay Differential Equations Using Multistage Optimal Homotopy Asymptotic Method," Journal of Mathematical and Fundamental Sciences, vol. 50, no. 3, pp. 221-232, 2018.

[2] N. Ratib Anakira, A. K. Alomari, and I. Hashim, "Optimal homotopy asymptotic method for solving delay differential equations," Mathematical Problems in Engineering, vol. 2013, Article ID 498902, 11 pages, 2013.

[3] A. K. Alomari, M. S. Noorani, and R. Nazar, "Solution of delay differential equation by means of homotopy analysis method," Acta Applicandae Mathematicae, vol. 108, no. 2, pp. 395-412, 2009.

[4] J. He, "Variational iteration method-a kind of non-linear analytical technique: Some examples," International Journal of Non-Linear Mechanics, vol. 34, no. 4, pp. 699-708, 1999. 
[5] J. He, "Variational iteration method-some recent results and new interpretations," Journal of Computational and Applied Mathematics, vol. 207, no. 1, pp. 3-17, 2007.

[6] J. H. He and X. H. Wu, "Variational iteration method: new development and applications," Computer \& Mathematics with Applications, vol. 54, no. 7-8, pp. 881-894, 2007.

[7] J. H. He, G. C. Wu, and F. Austin, "The variational iteration method which should be followed," Nonlinear Science Letters A : Mathematics, Physics and Mechanics, vol. 1, no. 1, pp. 1-30, 2010.

[8] N. Herisanu and V. Marinca, "A modified variational iteration method for strongly nonlinear problems," Nonlinear Science Letters A : Mathematics, Physics and Mechanics, vol. 1, no. 2, pp. 183-192, 2010.

[9] Z. M. Odibat and S. Momani, "Application of variational iteration method to nonlinear differential equations of fractional order," International Journal of Nonlinear Sciences and Numerical Simulation, vol. 7, no. 1, pp. 27-34, 2006.

[10] S. M. Goh, M. S. Noorani, and I. Hashim, "On solving the chaotic Chen system: a new time marching design for the variational iteration method using Adomian's polynomial," Numerical Algorithms, vol. 54, no. 2, pp. 245-260, 2010.

[11] Y. Molliq R, M. S. M. Noorani, and I. Hashim, "Variational iteration method for fractional heat- and wave-like equations," Nonlinear Analysis: Real World Applications, vol. 10, no. 3, pp. 1854-1869, 2009.

[12] M. A. Noor and S. T. Mohyu-Din, "Variational iteration method for solving higher-order nonlinear boundary value problems using Hes polynomials," International Journal of Nonlinear Sciences and Numerical Simulation, vol. 9, no. 2, pp. 141-156, 2008.

[13] G. Wu, "Challenge in the variational iteration method-a new approach to identification of the Lagrange multipliers," Journal of King Saud University - Science, vol. 25, no. 2, pp. 175-178, 2013.

[14] B. Batiha, M. S. M. Noorani, and I. Hashim, "Approximate analytical solution of the coupled sine-Gordon equation using the variational iteration method," Physica Scripta, vol. 76, no. 5, pp. 445-448, 2007.

[15] B. Batiha, M. S. M. Noorani, and I. Hashim, "Application of variational iteration method to a general Riccati equation," International Mathematical Forum, vol. 2, no. 56, pp. 2759-2770, 2007.

[16] G. Wu and D. Baleanu, "Variational iteration method for fractional calculus-a universal approach by Laplace transform," Advances in Difference Equations, vol. 1, pp. 1-9, 2013.

[17] A.-M. Wazwaz, "The variational iteration method for analytic treatment of linear and nonlinear ODEs," Applied Mathematics and Computation, vol. 212, no. 1, pp. 120-134, 2009.

[18] T. A. Biala, O. O. Asim, and Y. O. Afolabi, "A combination of the laplace transform and the variational iteration method for the analytical treatment of delay differential equations," International Journal of Differential Equations and Applications, vol. 13, no. 3, 2014.

[19] A. A. Elbeleze, A. Kılıçman, and B. M. Taib, "Homotopy perturbation method for fractional black-scholes european option pricing equations using sumudu transform," Mathematical Problems in Engineering, vol. 2013, Article ID 524852, 7 pages, 2013.

[20] Y. Liu and W. Chen, "A new iterational method for ordinary equations using sumudu transform," Advances in Analysis, vol. 1, no. 2, 2016.
[21] P. Goswami and R. T. Alqahtani, "Solutions of fractional differential equations by Sumudu transform and variational iteration method," The Journal of Nonlinear Science and its Applications, vol. 9, no. 4, pp. 1944-1951, 2016.

[22] F. B. M. Belgacem and A. Karaballi, "Sumudu transform fundamental properties investigations and applications," Journal of Applied Mathematics and Stochastic Analysis, vol. 2006, Article ID 91083, 23 pages, 2006. 


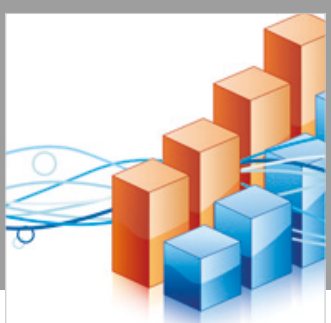

Advances in

Operations Research

\section{-n-m}
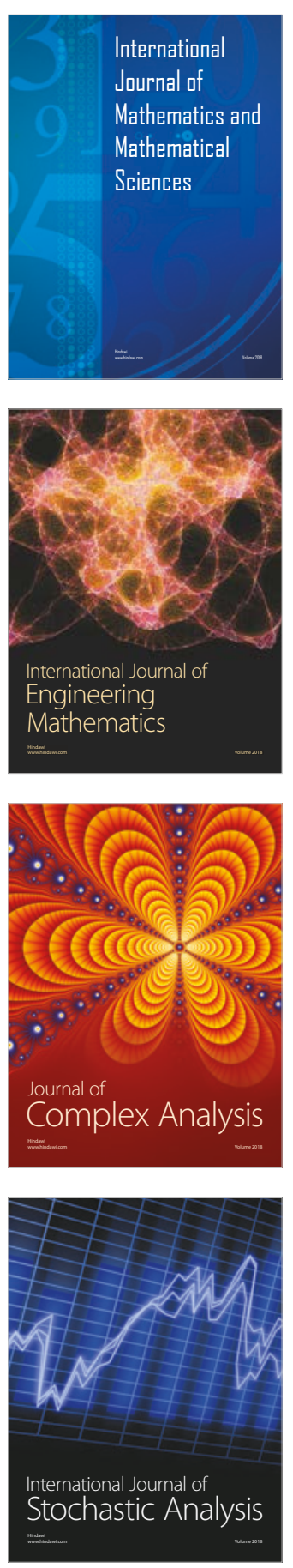
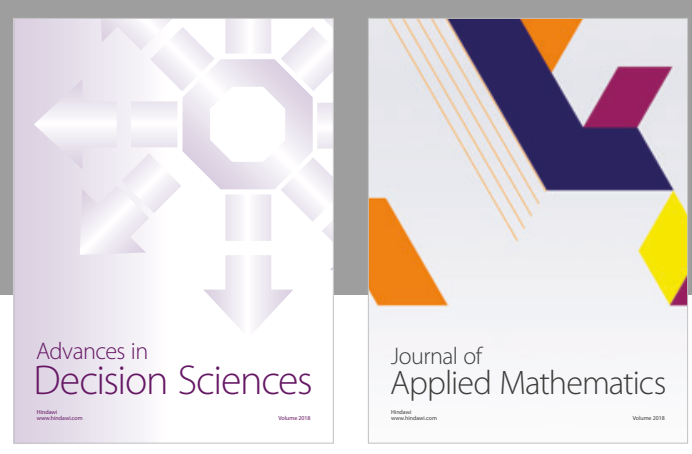

Journal of

Applied Mathematics
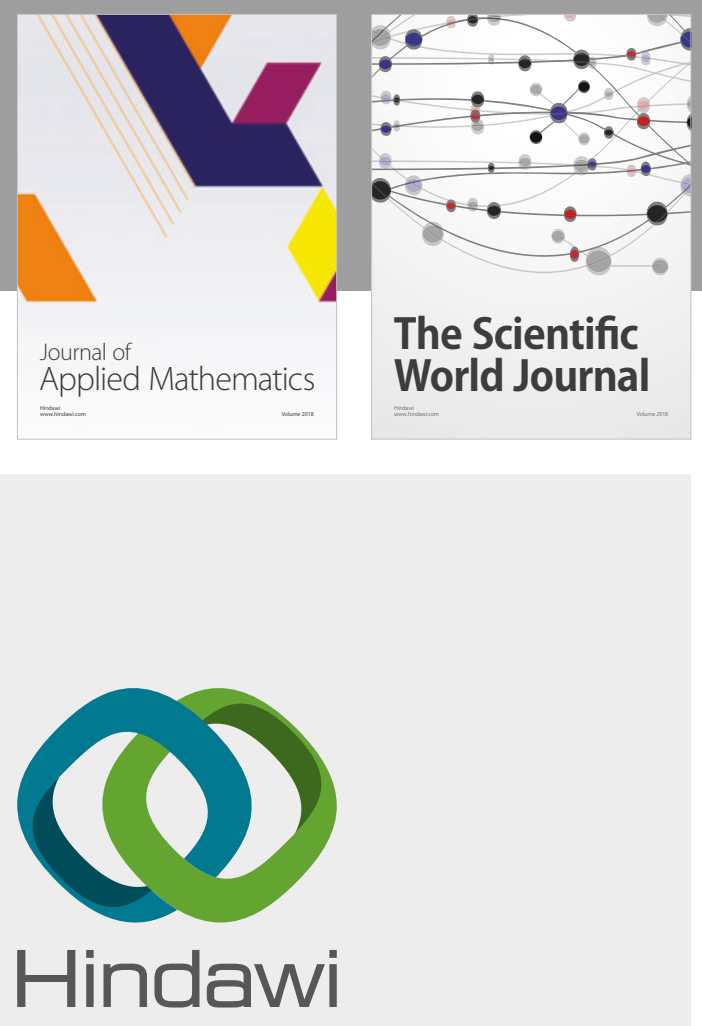

Submit your manuscripts at

www.hindawi.com

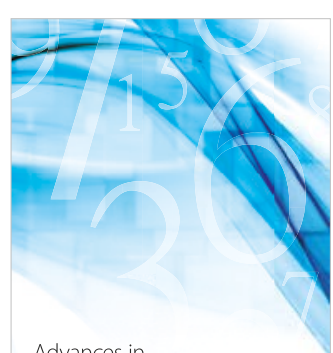

Advances in
Numerical Analysis
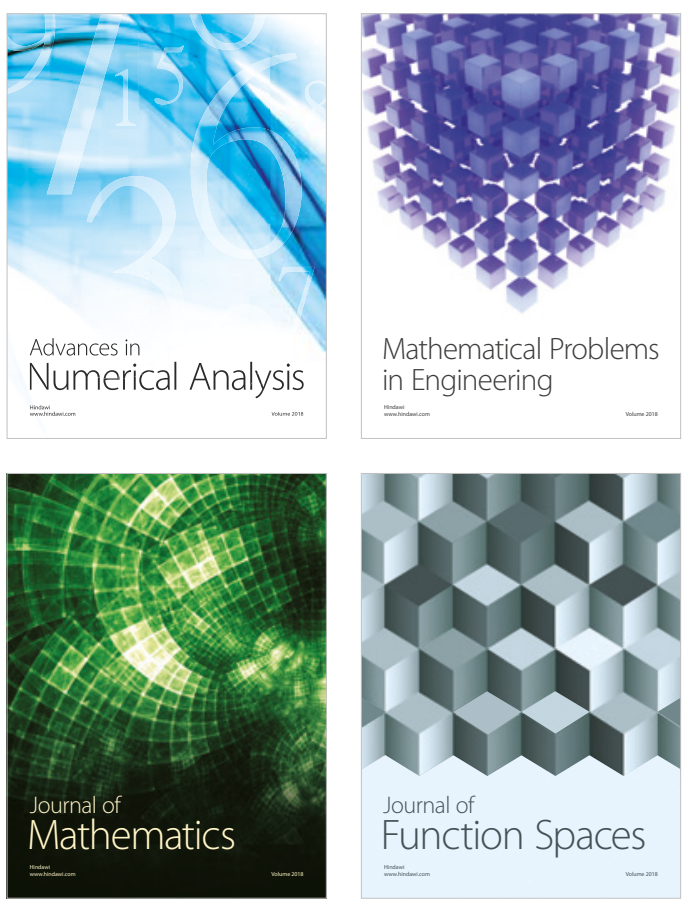

Mathematical Problems in Engineering

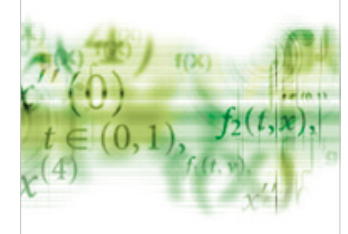

International Journal of

Differential Equations

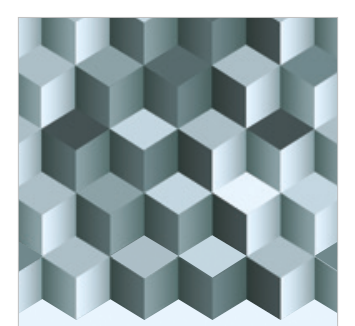

Journal of

Function Spaces

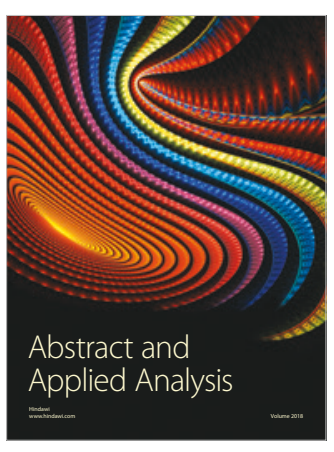

The Scientific

World Journal

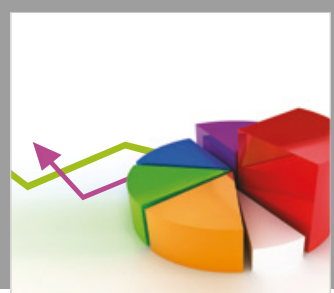

Journal of

Probability and Statistics
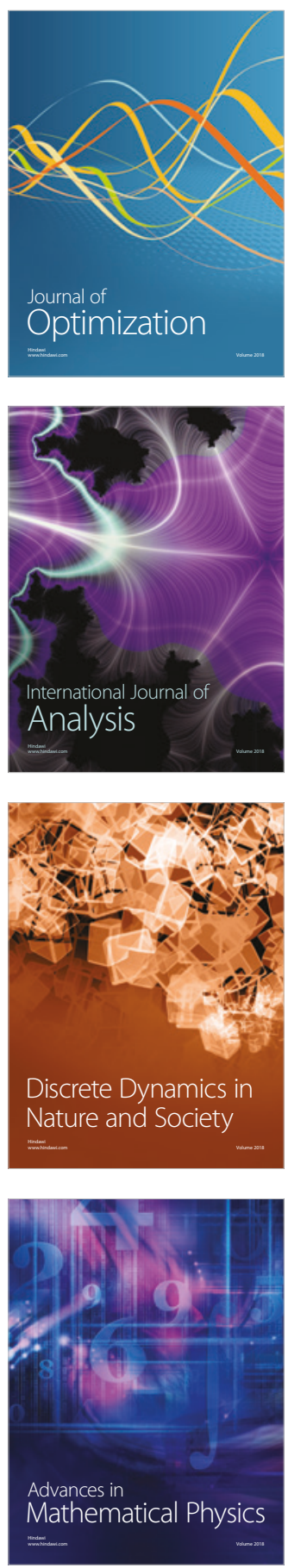